\title{
Fairness and Physical Layer Capture in Random Access Networks
}

\author{
Hoon Chang \\ Department of Computer Science \\ Columbia University \\ New York, NY 10027 \\ Email: hchang@cs.columbia.edu
}

\author{
Vishal Misra \\ Department of Computer Science \\ Columbia University \\ New York, NY 10027 \\ Email: misra@cs.columbia.edu
}

\author{
Dan Rubenstein \\ Department of Electrical Engineering \\ Columbia University \\ New York, NY 10027 \\ Email: danr@ee.columbia.edu
}

\begin{abstract}
While physical layer capture has been observed in real implementations of wireless devices which randomly accessing shared channels, fair rate control algorithms based on accurate channel models describing the phenomenon have not been developed. In this paper, using our general physical channel model, we formally present the characteristics of the feasible domain of a general fairness problem and utility fairness under physical layer capture. We show that the allocation domain is not convex and the previous optimization schemes cannot be applied. We further show the objective function for utility fairness is concave on the domain of channel access attempt probability. We clarify that the optimal attempt probability of the node in the log utility fairness is proportional to its interference effect on its neighbors. Based on our analysis, we propose a rate determination algorithm to achieve logutility fairness for random access networks. Running in a fully distributed manner, our rate control algorithm can reach an assignment close to the optimum, in contrast of the previous algorithms causing node starvation in presence of physical layer capture. The accuracy of our algorithm is verified through simulations and our results indicate the accuracy error for log utility fairness is less than $5 \%$ for $97 \%$ of the nodes.
\end{abstract}

\section{INTRODUCTION}

While physical layer capture has been reported to cause unfair allocations in random access networks [1], fair scheduling has been only studied in literature with unrealistic wireless models. Traditional modeling of random access networks [2], [3], [4] assumes that transmissions are not corrupted if and only if all other nodes within certain interference ranges centered in the sender and receiver are not transmitting frames. Transmissions

This material was supported in part by the National Science Foundation under CAREER Award No. CNS-560153, ANI-0238299 and by support from Samsung, the Intel Information Technology Research Council, and Microsoft Research. Any opinions, findings, and conclusions or recommendations expressed in this material are those of the authors and do not necessarily reflect the views of the National Science Foundation. outside of the range are assumed not to affect the transmission at all. With this assumption, conflict graphs can be formed and simplify analysis on access protocols.

In reality, this assumption generally turns out to be false. A transmitted frame can get through a collision and be received at the receiver without error, if the total interference strength is under some threshold. The phenomenon is referred to as physical layer capture [5]. However, as the number of simultaneous transmissions increases, the cumulative interference from the neighbors may exceed the threshold and corrupt frame reception at the receiver even when the neighbors may not interfere in isolation.

The principal problem with prior work is the assumption of static conflict graphs. Assuming interference ranges, only nodes within the ranges are individually considered to have conflict relationships. However, in presence of physical layer capture, the cumulative interference from a given set of transmitting nodes may or may not cause conflicts and this makes the conflict graph dynamic. That is, simultaneous transmissions from specific sets of nodes, a probabilistic occurrence, causes new conflict relationships to come and go. Thus, the conflict graph, especially in dense networks, is constantly changing thereby frequently nullifying any optimality/fairness results derived with static-conflict graphs.

Fair allocation algorithms proposed so far rely on the simplification to physical channel models. In [6], Nandagopal et al present a fully distributed rate control algorithm depending only on the loss rate at the receiver, assuming the effect of interference is completely symmetric. The algorithm in [7], built using the idea of interference ranges, quickly determines optimal attempt probabilities based on the number of neighbors; however, the effect of cumulative interference signals is not considered at all. In presence of physical layer capture, these algorithms inaccurately estimate fair throughputs potentially causing biased allocations. 
The goal of this work is to formally investigate fairness under the effect of physical layer capture in random access networks. Our major contributions are:

- We first present the general characteristics of fair bandwidth allocations for random access networks in presence of physical layer capture. We formally prove the feasible region of bandwidth allocations in physical layer capture is non-convex. We further discuss that the convex conversion technique proposed in the previous work cannot be applied in physical layer capture.

- Next, we investigate log utility fairness under physical layer capture. Our analysis demonstrates that log-utility fairness allows the sender to access the channel at a probability given by its interference effect to its neighbors. We prove that the aggregate utility is a concave function of attempt probabilities and present a distributed algorithm to assign attempt probabilities for log-utility fairness.

- Finally, our algorithm is verified through extended simulations. Our algorithm significantly improves the minimum node throughput as well as the aggregate utility. The simulation results indicate that previously proposed algorithm based on conflict graphs may cause starvation. Ignoring the effect of cumulative interference, the previous algorithm tends to assign attempt probabilities higher than the optimum and make about $22 \%$ of nodes in our simulations starve due to frequent interference.

In this paper, we focus on dense wireless networks like in offices and urban residential areas. In such networks, one or few terminals are close to an access point and tend to capture strong signals from it. The dense distribution of access points and terminals, however, enlarges the effect of cumulative interference on frame reception.

For MAC protocol, slotted Aloha is considered. Note that our analysis and fair scheduling algorithm for slotted Aloha can be extended for CSMA/CA protocols with a single carrier-sensing range. In CSMA/CA fair scheduling, if the channel is idle, nodes access the channel per time slot with probabilities determined by scheduling algorithms. Assuming all nodes are transmitting fixedlength frames and located within a single sensing range, the nodes are fully synchronized and the difference from slotted Aloha is limited to the length of idle slots. Our extended work for CSMA/CA can be found in [8] and analysis with multiple ranges is left for the future.

The rest of our paper is structured as follows: section II reviews related work. In Section III, our system model and the characteristics of the feasible allocation region are presented. Section IV investigates utility fair- ness in presence of physical layer capture and presents our distributed rate control algorithm. Section V shows simulation results of our fair scheduling algorithm. Section VI draws the conclusions of this study. All necessary proofs are provided in the appendix.

\section{RELATED WORK}

After investigation by Soroushnejad and Geraniotis [5], slotted-Aloha systems with the effect of physical layer capture have been extensively studied. Namislo analyzes the effect of physical layer capture in [9]. Cidon et al in [10] investigate the behavior of various collision resolution algorithms with the capture effect. However, their analysis and models are limited to networks with a single receiver and multiple senders. The authors of [11], [12] present analytical models for multi-hop networks, but their work assumes that nodes are spatially distributed according to a Poisson process and only captures the average throughput over all Poisson configurations.

Analyzing random access protocols in wireless networks, [3] provides one of the first analytical models where interference ranges are used to identify asymmetric interference. However, without considering cumulative interference signals, the authors suppose that only multiple transmissions within the ranges destroy the frames involved. While this assumption carries over to recent analysis work [2], [4], we have presented the first analytical model of CSMA/CA random access protocols in presence of physical layer capture [13]. We formulate a complete expression for the error probability with the capture effect. Using the least squares method and fixed point iteration, our analysis obtains accurate expectation on error probabilities and throughputs of nodes. The prediction error for error probability is less than $10 \%$ for more than $98 \%$ of nodes.

Rate control algorithms for fairness have been developed based on models using interference ranges. For proportional fairness [14], Kar et al [7] show that optimal attempt probabilities are given by the number of neighbors in a conflict graph. In [6], Nandagopal et $a l$, assuming interference relation is symmetric, develop a general objective function penalizing the sender in proportional to the local loss rate. However, in presence of asymmetric interference, the algorithm punishes nodes that are vulnerable to interference rather than nodes causing interference.

Wang et al, in [15], [16], propose a distributed algorithm for utility and max-min fairness using the standard dual approach with conflict graphs. Recent research for max-min fairness also uses interference ranges and conflict graphs as in [17], [18]. In real environments, 
under the effect of frame capture, the proposed algorithms cannot achieve optimal bandwidth allocations since interference is asymmetric and cumulative.

\section{SySTEM MODEL}

\section{A. General Physical Channel Model}

In this paper, we consider link layer fairness with multiple pairs of a sender and receiver. Senders and receivers are stationary and randomly placed in an area. Each sender always has frames to send and access the channel randomly in each slot. After receiving error-free frames, receivers immediately acknowledge their sender.

Assuming slotted-Aloha systems, the node throughput is a function of the attempt rate and successful transmission probability. The attempt rate is determined by the node itself but the success of a transmission depends on the activity of neighbor nodes.

The successful reception of a transmitted frame is determined through two significant stages. Initially, the frame must be detected by the receiver. Following this, the frame must be successfully received in the presence of interference both from other transmissions and external noise sources. In literature, many models for these two stages have been proposed [19], [20], [21].

Rather than using specific capture models, we introduce capture function $c_{i}(J)$ to generalize. Given a node set $J$, that does not include node $i$, the function computes the successful detection-and-reception probability of node $i$ 's transmitted frames, in conforming to a specific capture model. The capture function is defined to comply with any capture models and work in consideration of interference signals from all nodes in $J$.

With capture function $c_{i}(J)$, the success transmission probability $q_{i}$ and node throughput $x_{i}$ of node $i$ are described by:

$$
\begin{aligned}
q_{i} & =\sum_{J \subset N_{i}} c_{i}(J) \prod_{j \in J} f_{j} \prod_{k \in N_{i}-J}\left(1-f_{k}\right), \quad \text { and } \\
x_{i} & =f_{i} q_{i}=f_{i}\left(\sum_{J \subset N_{i}} c_{i}(J) \prod_{j \in J} f_{j} \prod_{k \in N_{i}-J}\left(1-f_{k}\right)\right),
\end{aligned}
$$

where $N_{i}=N-\{i\}, N$ is the entire set of nodes and $f_{j}$ is the per-slot transmission probability of node $j$.

Note that we do not consider channel fading here, assuming all nodes in the network are stationary. Although channel fading would cause the received signal power to fluctuate, the incoming signal strength is assumed to be approximately constant for stationary nodes at a given power level, as observed in [22].
Now we present a simple example that depicts the problems of an access rate allocation based on staticconflict graphs. Figure 1 shows an example of the Flowin-the-Middle (FIM) problem [4] where a flow in the middle of two others could starve. Node $i, j$ and $k$ are placed mutually apart and any two signals from them do not interfere with each other (as in time slot $A$ to $D)$. However, simultaneous transmissions from $j$ and $k$ causes frame corruption to node $i$ (in time slot $E$ ). In our general model, $c_{i}$ is zero if $J=\{j, k\}$ and one otherwise. $c_{j}$ and $c_{k}$ are equal to one.

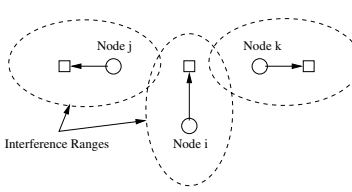

(a) Node Placement

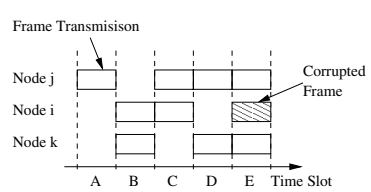

(b) Channel Access
Fig. 1. Flow-in-the-Middle Example.

In the traditional modeling, the static-conflict graph is formed with vertices for node $i, j$ and $k$ and has no edges since there are no peer nodes within interference ranges. As the conflict could not be identified in the graph, all nodes in Figure 1 would be allowed to transmit frames all the time for any given fairness. This would make node $i$ starve and result in an unfair bandwidth allocation.

\section{B. General Fairness Problem and Feasible Region}

In this section, we formulate a general fairness problem and show that the feasible region is not convex. In literature, a general fairness problem is viewed as a mathematical optimization problem with constraints. For any fair allocations, the general fairness problem, $P$ is formulated by:

$$
\begin{aligned}
& P: \max _{i} g\left(y_{i}\right) \\
& \quad y_{i} \leq x_{i}=f_{i} q_{i}
\end{aligned}
$$

The objective function $g$ is determined for given fairness. For example, $g$ is the aggregate utility $\left(\sum_{i} \log \left(y_{i}\right)\right)$ in log-utility fairness. For max-min fairness, $g$ is given by $-\frac{1}{2} \sum_{i}\left(\log \left(y_{i}\right)\right)^{2}$ with additional constraints where $y_{i} \leq$ $y_{s}$ for all neighbor $s$ [16]. In this general formulation, a fairness problem is to seek the values of the variables $y_{i}$ from within an allowed region of node throughputs to maximize the objective function.

We now show that the feasible allocation region for $y_{i}$ is not convex. Define $J$ as an interference set of $i$ if $c_{i}(J)<1$. Set $J$ is referred to as a minimum interference 

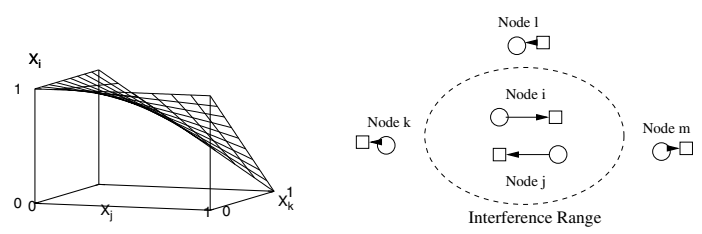

Fig. 2. Non-Convex Region. Fig. 3. 5-Node Example.

set of $i$ if $c_{i}$ of any proper subset of $J$ is one. Our nonconvexity theorem is presented as follows:

Theorem 1 (Non-Convexity of Feasible Region): if there exists node $i$ and its minimum interference set, $I$ in the network such that the size of $I$ is greater than one, the feasible region of node throughputs is non-convex.

The proof is presented in Appendix.

For the example in Figure 1, the feasible throughput region, plotted in Figure 2, is non-convex since the minimum interference set of node $i$ is $\{j, k\}$.

Theorem 1 indicates that the powerful tools of convex analysis are not applicable for fairness in presence of physical layer capture. In the previous channel models with interference ranges, constraints for $y_{i}$ are described as convex by using maximal cliques on conflict graphs [6] or the conversion techniques presented in [16], [15]. The authors of [16], [15] also show that the converted convex optimization problems are easily solved by the dual and primal approaches. However, due to the dynamicity of conflict graph, cliques in presence of physical layer capture would not describe all contentions among nodes. On the other hand, transformed constraints for $y_{i}$ by the techniques in [16], [15] are not convex if the effect of cumulative interference is significant. We prove this by a counter example. Consider the example in Figure 1. Applying the transforming techniques to the constraints in general problem $P$, the logarithm of $y_{i}$ must be less than or equal to the sum of $\log \left(f_{i}\right)$ and $\log \left(q_{i}\right)$. However, the logarithm of $q_{i}$, equal to $\log \left(1-f_{j} f_{k}\right)$ in this example, is neither convex nor concave function of $f_{j}$ and $f_{k}$. It then follows that the equivalent problem is not convex.

Non-convex optimization problems, like the general fairness problem, are generally hard. Simply applying the convex optimization techniques proposed in [23], [15] tends to fall into the extreme points of the feasible region and reach unfair stable states. Instead, we present our fast algorithm for log-utility fairness in Section IV.

\section{Max-Min and Log Utility Fairness}

In this section, we compare max-min and log-utility fairness. Max-min fair scheduling generally obtains lower aggregate throughput with asymmetric interference. We first present the following theorem for max-min fairness in presence of physical layer capture:

Theorem 2 (Throughput in Max-Min Fairness): for the max-min fairness, the throughput of a node interfering is at most them of its interfered neighbors.

Even when cumulative interference from multiple neighbors does not occur frequently, throughput of the interfering neighbors would drop for max-min fairness. Figure 3 shows this example. Assuming simultaneous transmissions from node $k, l$ and $m$ interfere with node $i$ and $j$, all the nodes have the same throughput as that of the other nodes, $i$ and $j$ in max-min fairness.

However, for log-utility fairness, the throughput of outer nodes as well as the total throughput is boosted by small reducing inner nodes' throughputs. In this example, the log-utility fair allocation achieves aggregate throughput almost twice that of max-min fairness [24]. Note that the denser the network is, the more interfering outer nodes like node $k$ to $m$ may exist; the throughput enhancement can be larger. This property makes logutility fairness, well known as a robust trade-off between fairness and efficiency [25], more preferred in presence of physical layer capture. More details on our choice of log-utility fairness can be found in [24].

\section{LOG-UTILITY FAIR SCHEDULING}

\section{A. Concaveness of Utility Function}

Considering cumulative interference, the log utility function is neither convex nor concave since the domain of node throughputs is not convex. However, the log utilities on the domain of attempt probabilities, which is $|N|$-dimensional space, are always concave. We present the theorem of concaveness as follows:

Theorem 3 (Concaveness of Log Utilities): the sum of $\log$ utilities on the domain of attempt probabilities in presence of physical layer capture is concave.

This property is also valid for general utilities as in the following corollary. Note that the general form of utility functions is $U_{i}(F)=-1 / x_{i}^{v}$ for attempt probability vector $F$ and $v>0$. When $v=1$, the utility function characterizes the minimum potential delay fairness model [26]. For the special case when $v=0$, the utility function is given by $U_{i}(F)=\log \left(x_{i}\right)$.

Corollary 1 (Concaveness of General Utilities):

the sum of general utilities on the domain of attempt probabilities in physical layer capture is concave.

The concaveness property allows to use partial derivatives for the optimal allocation. Since the general utility is differentiable and concave, partial derivatives with respect to attempt probabilities should be zero at the 
maximum. Furthermore, if each node individually tries to make its partial derivative zero by changing its attempt probability, the optimal point is obtained, as follows:

Theorem 4 (Convergence): if each node adjusts its attempt probability in order to satisfy the condition where the partial derivative is zero, the system converges to the optimal allocation point maximizing the aggregate utility.

Based on Theorem 4, we present our novel distributed algorithm for log utility fairness in the next sections.

\section{B. Formulation for Optimal Attempt Probability}

We first formulate the optimal attempt probability of node $i$ maximizing the aggregate utility. Let $S=$ $\sum_{i} \log \left(x_{i}\right)$. The partial differentiation of $S$ with respect to the attempt probability $f_{i}$ of node $i$ is written as:

$$
\frac{\partial S}{\partial f_{i}}=\frac{1}{f_{i}}-\underbrace{\sum_{j} \frac{q_{j \mid \bar{i}}-q_{j \mid i}}{q_{j}}}_{A}=0,
$$

where $q_{j \mid i}$ is the conditional probability of successful transmissions from node $j$, given node $i$ is transmitting frames in the same slot. $q_{j \mid \bar{i}}$ is the conditional successful probability of node $j$ when node $i$ is transmitting no frame. Note that $q_{i \mid i}=q_{i}$ and $q_{i \mid \bar{i}}=0$ by definition.

Since $q_{j}=\left(1-f_{i}\right) q_{j \mid \bar{i}}+f_{i} q_{j \mid i}$ and $q_{j \mid \bar{i}}-q_{j \mid i}$ equals $\left(q_{j}-q_{j \mid i}\right) /\left(1-f_{i}\right)$, Equation 3 is simplified and the optimal attempt probability $f_{i}^{*}$ satisfies the following:

$$
\frac{1-f_{i}^{*}}{f_{i}^{*}}=\sum_{j}\left(1-\frac{q_{j \mid i}^{*}}{q_{j}^{*}}\right) .
$$

Equation 4 is interpreted as follows: at the optimal state, the sender is charged a price equal to the sum of its interference effect to neighbors, to access the channel. The effect of interference to neighbor node $j$ is here defined as $\left(1-q_{j \mid i}^{*} / q_{j}^{*}\right)$, gauging how much the transmissions of $j$ are degraded by node $i$. The price is paid with the average number of waiting slots $\left(1 / f_{i}^{*}-1\right)$. Note that ignoring the effect of cumulative interference, $q_{j \mid i}^{*} / q_{j}^{*}$ in the equation is either zero (if $i$ is within the interference range of $j$ ) or one (otherwise) and the number of waiting slots in Equation 4 becomes equivalent to the number of neighbors in the interference ranges, as shown in the previous model [7].

\section{Obtaining Optimal Point}

We provide an algorithm for optimal rates using the fixed-point iteration. Let $F^{*}$ be the optimal attempt probability vector satisfying Equation 3 and function $f\left(F^{*}\right)=F^{*}$. Since function $f$ is continuous and maps a rate vector to another, $f$ has a fixed point (Brouwer's fixed point theorem [27]). We can further show that $f$ converges to the fixed point as an immediate consequence of Brouwer's theorem and Theorem 4. Thus, if we know $f, F^{*}$ is obtained by continuously applying $f$.

Now, we formulate function $f$ satisfying Equation 3 . Assume that for all $j, q_{j \mid i}$ and $q_{j}$ are known. To make the equation simple, we approximate Part $\mathrm{A}$ in Equation 3 to a linear function of $f_{i}$ as follows:

$$
\text { Part } \mathrm{A} \approx a f_{i}+b .
$$

Recall that Part A depends on $f_{i}, q_{j \mid i}, q_{j \mid \bar{i}}$ and $q_{j}$ but $q_{j}$ can be computed from the others. With known $q_{j \mid i}$ and $q_{j \mid \bar{i}}$, we obtain $a$ and $b$ by applying the least squares method. Varying $f_{i}$, we uniformly sample $K$ points from the curve of Part A and find a linear function that closely approximates the sampled data. The approximation error is very small in most of cases; the square of the residuals is at least 0.968194017 for typical values of $f_{i}^{*}$. Details are found in [28]. After linear approximation, we have a quadratic formula $1 / f_{i}=a f_{i}+b$ from Equation 3 and $f_{i}$ is finally given by:

$$
f_{i}= \begin{cases}\min \left(\frac{-b+\sqrt{b^{2}+4 a}}{2 a}, 1\right) & (\text { if } a>0) \text { and } \\ \min \left(\frac{1}{b}, 1\right) & (\text { if } a=0),\end{cases}
$$

where $\min (\infty, 1)=1$. The total computation time of $f_{i}$ is $O(K \cdot|N|)$, where $K$ is the number of sample points.

We show the solution is always unique. Since Part A in Equation 3 is a strictly increasing function of $f_{i}$, any lines connecting any two points on the curve of Part A have a positive slope (i.e., $a$ of Equation 5 is always positive); linear functions with slope $a>0$ have only a single common point with inverse function $1 / f_{i}$.

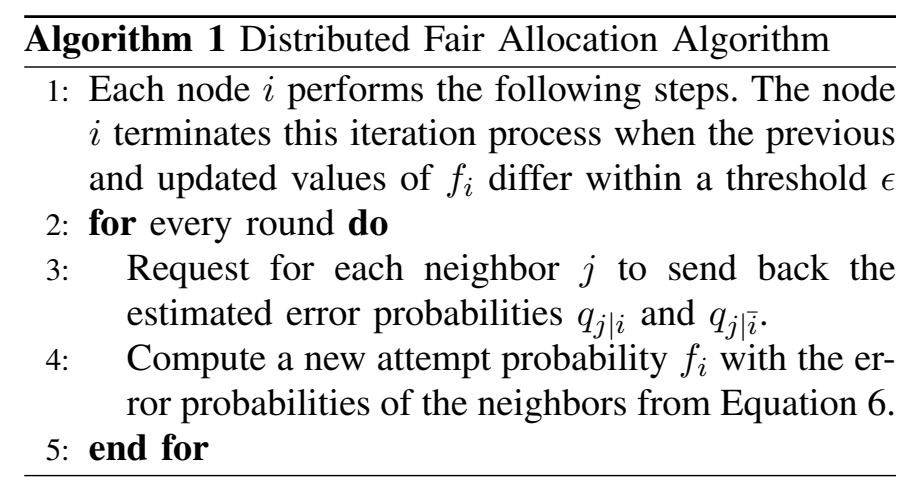

With function $f$ (i.e., Equation 6), we implement Algorithm 1 in a distributed way to achieve log utility fairness. Assuming node $i$ knows neighbors that it interferes with (i.e., nodes whose successful transmission probability is affected by the behavior of $i$ ), nodes communicate with the neighbors over wired or wireless links. 
TABLE I

OPERATION PARAMETERS

\begin{tabular}{|c|c|c|c|}
\hline \multicolumn{2}{|c|}{ Physical Layer } & Ant. Efficiency & 0.8 \\
\hline Frequency & $5 \mathrm{GHz}$ & Antenna Loss & $0.5 \mathrm{~dB}$ \\
\hline Path Loss Model & Two Ray & Antenna Height & $1.5 \mathrm{~m}$ \\
\hline Shadowing Model & Constant & MAC La & \\
\hline Shadowing Mean & $4 \mathrm{~dB}$ & MAC Protocol & Slotted \\
\hline Fading Model & None & & Aloha \\
\hline Temperature & $290 \mathrm{~K}$ & Slot Time & $177 \mu \mathrm{s}$ \\
\hline Noise Factor & 7 & Tx Time & $177 \mu \mathrm{s}$ \\
\hline Tx Power & $16 \mathrm{dBm}$ & Tx Speed & 54Mbps \\
\hline Rx Sensitivity & $-82 \mathrm{dBm}$ & MAC Header & 28 bytes \\
\hline Antenna & Omni. & ACK Frame & 14 bytes \\
\hline
\end{tabular}

Note that access points in corporate and residential areas are typically connected to a wired local area network and can often cooperate with each other. The information of neighbors can be entered by the network administrator.

In Algorithm 1, each node updates its attempt probability until it converges; nodes stop the process when the difference of the updated probability is less than a threshold. To calculate their new attempt probabilities, nodes need to know the following: the error probabilities $q_{j \mid i}$ and $q_{j \mid \bar{i}}$ of neighbor $j$, as in Equation 5.

To obtain $q_{j \mid i}$ and $q_{j \mid \bar{i}}$, node $i$ only needs to request interfered neighbors whose $q_{j \mid i}$ is not zero. If node $j$ is close enough, collisions occur with the node $i$ and $q_{j \mid i}=0 . q_{j \mid i}$ equals to $q_{j}$ and the term for node $j$ in Part A is always one regardless of the value of $f_{i}$. Thus, node $i$ requests the limited number of neighbors and all information for rate computation is locally obtained.

As the final remark, $q_{j \mid i}$ and $q_{j \mid i}$ can be measured by real time. For $q_{j \mid \bar{i}}$, whenever needed, node $i$ holds off its transmissions and ask the neighbors to measure the successful transmission probability, which is $q_{j \mid \bar{i}}$. Obtaining $q_{j \mid i}$ is not simple since neighbors do not know when node $i$ is transmitting. Instead, node $i$ requests the current success probability of transmission to neighbors. Then, $q_{j \mid i}$ can be computed from the measured $q_{j \mid \bar{i}}$, the probability and the current value of $f_{i}$.

\section{Simulation Results}

\section{A. Simulation Parameters}

To verify our algorithm, pairs of a sender and receiver are randomly placed in an area $100 \times 100 \mathrm{~m}^{2}$. The distance between a sender and receiver is selected in a random way but the maximum is set to 30,10 and 5 meters respectively. 10 different random placements are considered for each number of node pairs.

Our simulator is based on Qualnet simulator, which provides a more accurate channel model than ns-2 [29].
Table I shows the operation parameters. The transmitter is in half-duplex mode and operating in the 802.11a 5$\mathrm{GHz}$ channel. From locations of nodes, we compute the strength of signals using the two-ray path loss model and the BER (bit error rate) of the received frame is obtained from calculation of Qualnet. Receiving an uncorrupted frame, the receiver always responds with an acknowledgment.

MAC protocol is slotted Aloha. Nodes are synchronized and channel accesses occur at every slot. The attempt probability is determined by a given fair algorithm, which is adjusted from the frame error probability.

\section{B. Minimum Interference Set}

We first investigate the size of minimum interference sets. Varying the number of nodes from 6 to 16, we measure minimum interference sets of all nodes. Figure 4 shows a node placement example with the total 6 node pairs. Here, all odd-numbered nodes are senders, which are connected in the figure with corresponding receivers. The maximum distance is 10 meters in this example.

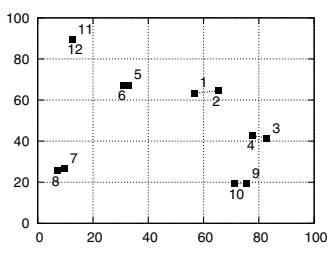

(a) Node Placement

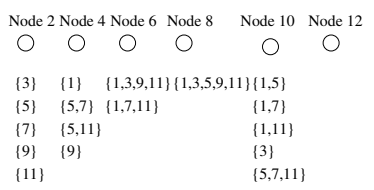

(b) Interference Sets
Fig. 4. 6 Node-Pair Example.

Figure 4(b) shows the minimum interference sets of the receivers. The interference sets are displayed under the corresponding receivers. For example, Receiver node 2 , placed $8.65 \mathrm{~m}$ away from its sender Node 1 , has 5 minimum interference sets, $\{$ Node 3$\},\{$ Node 5$\},\{$ Node $7\}$, \{Node 9\} and \{Node 11\}. However, Receiver 4 has two single-node sets and two two-node sets. Since the receiver is $5.23 \mathrm{~m}$ away, shorter than the distance of Receiver 2, and located further right in the area, Node 5, 7 and 11 cannot interfere in isolation. Note that Receiver 12 does not have an interference set.

In Figure 5, we show the statistics of all minimum interference sets for 6 to 16 node pairs in our simulations. With 6 node pairs and the maximum distance $30 \mathrm{~m}$, which is denoted as ' $(30,6)$ ' in the figure, most of the minimum interference sets have a single interferer. The black portion of the histogram indicates the percentage of interference sets including only a single interferer, and 


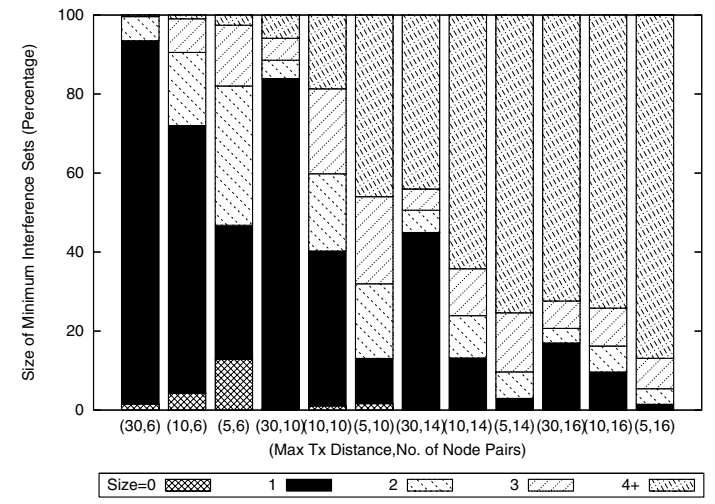

Fig. 5. Minimum Interference Sets for Total 1,380 Node Pairs.

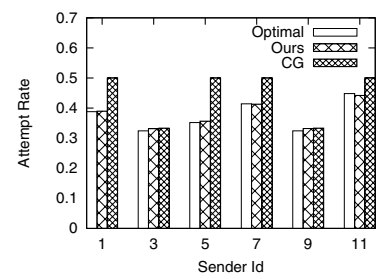

(a) Attempt Rate

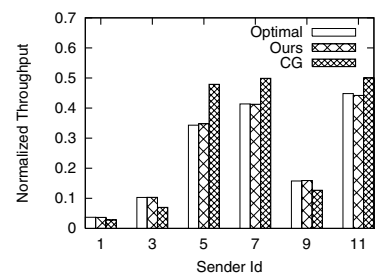

(b) Throughput
Fig. 6. Optimality in 6-Pair Example.

hence prior work on static-conflict graphs is applicable. However, as either the node density increases or the distance becomes shorter, the percentage of single-node interference sets decreases. In these scenarios, dynamicconflict graphs come into play and our algorithm becomes more accurate than prior work.

\section{Optimality Test}

The performance of our algorithm is compared with the algorithm using conflict graphs [7]. The algorithm in the previous work is referred to as $C G$. The NewtonRaphson method [30] is used to obtain the optimum. Note that unlike ours, the Newton-Raphson method is not suitable for distributed scheduling since it uses the Hessian matrix of the aggregate utility formed from the information of all nodes' transmission errors.

Figure 6 shows the results from the previous example. Compared to the optimum, algorithm $C G$ allows nodes to transmit frames with higher rates. Since the algorithm does not consider nodes outside of interference ranges, it underestimates the transmission error probabilities and assigns higher attempt probabilities. For example, Node 5 and 7 are allowed high-rate transmissions since they belong only in Node 2's interference range. However, al- lowing higher attempt probabilities degrades the throughput of nodes whose link quality is not good. Nodes with many minimum interference sets, like Node 3 and 9, suffer from high transmission errors. This may cause node starvation, worsening the problem of unfairness.

Figure 7 shows the entire results from our simulations. The total number of the simulations is 180 and the number of node pairs runs from 6 to 16. Given a number of nodes, we plot in Figure 7(a) the average difference of the aggregate utility between ours and algorithm $C G$ 's. Note that we compute the base-10 logarithm and set the maximum and minimum attempt probabilities to 0.9999 and 0.001 to prevent complete node starvation.

With the maximum distance $30 \mathrm{~m}$, the $\log$ utility is not much improved. However, as the maximum distance becomes short and the capture probability increases, the aggregate utility significantly increases by up to 10 . Furthermore, in algorithm $C G$, several nodes is starved due to the aggressive attempt probability allocations. More than $22 \%$ of the nodes starve with a throughput less than 0.001 with the maximum distance $5 \mathrm{~m}$.

While $\log$ utility is an implicit measure of performance, we now depict tangible improvements in the functioning of the network. Figure 7(b) shows the ratio of the minimum node throughputs in ours and algorithm $C G$ 's, excluding the starved nodes that have throughput less than 0.001. The maximum distance is $5 \mathrm{~m}$. Even discounting starved ones, our algorithm allows a higher throughput to nodes with low-quality links. The minimum node throughput in the network is improved by around 5 times on average with the maximum distance $5 \mathrm{~m}$. In the entire simulations, the minimum node throughput except that of starved nodes increases by more than 3.18 times on average. Note that the total throughput is not affected so much. The total throughput decreases by $7 \%$ with the maximum distance $5 \mathrm{~m}$ and $5 \%$ on average of the all simulation results.

To measure fairness in terms of node throughput, the Jain's fairness index [31] is presented in Figure 7(c). While our algorithm achieves stable throughput fairness, the algorithm $C G$ worsens the unfairness as the effect of physical layer capturing increases.

In Figure 8(a) and 8(b), we show the accuracy of our algorithm, given by the difference divided by the optimum. In total 1,980 rate computations, our accuracy error is less than $5 \%$ for more than $97 \%$ of nodes. Using algorithm $C G$, more than $55 \%$ of the total nodes are assigned attempt rates $20 \%$ larger than the optima.

The convergence time of our algorithm is also investigated. Our algorithm is run until the difference between the previous and updated attempt probabilities 


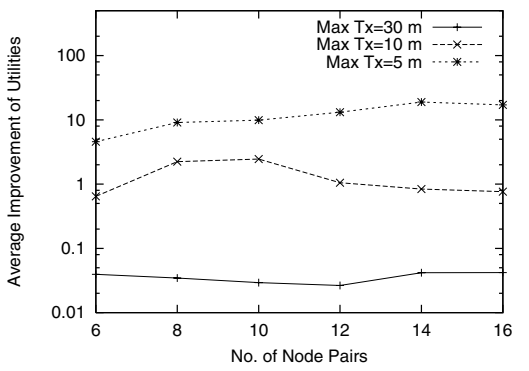

(a) Log Utility

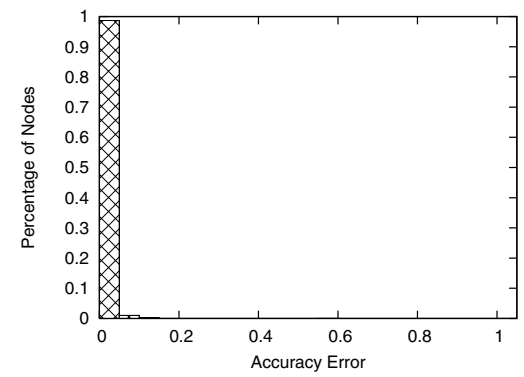

(a) Our Algorithm

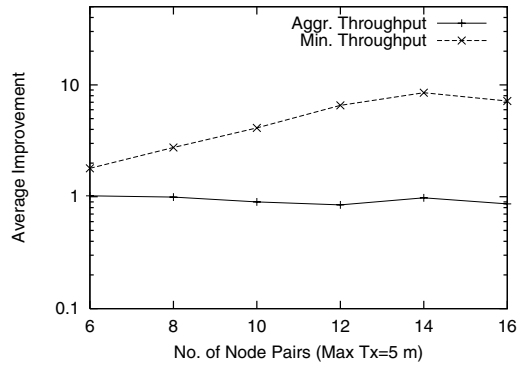

(b) Throughput Enhancement

Fig. 7. Utility Improvement.

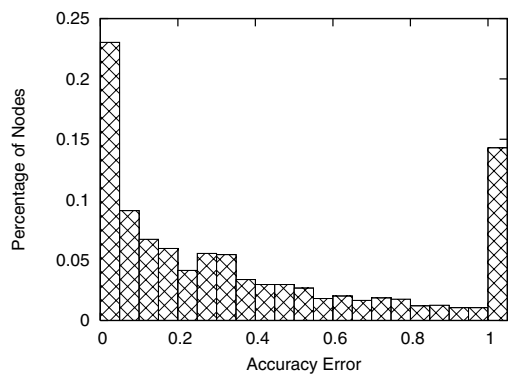

(b) Algorithm $C G$

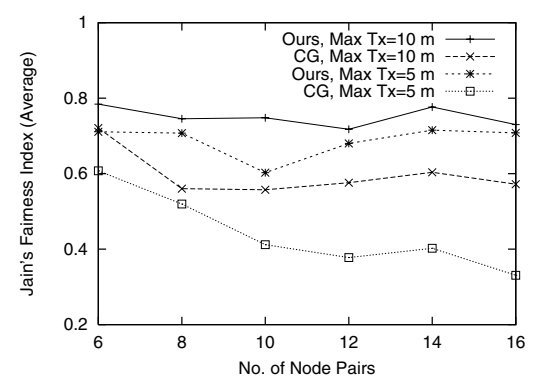

(c) Fairness Index

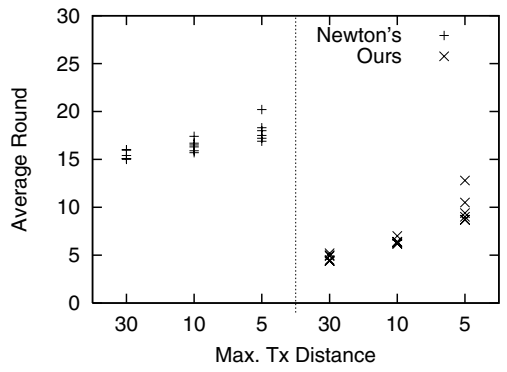

(c) Convergence Time

Fig. 8. Accuracy of Assigned Attempt Probabilities and Convergence Time.

is less than $10^{-7}$. Time is measured in unit of processing rounds. As shown in Figure 8(c), the convergence time is related to the topology of the network rather than the number of nodes. With the same maximum distance, varying the number of nodes does not significantly change the results. We also plot the convergence time of the Newton-Raphson method for comparison. Even though the method can be improved by using the line search, the computation time with a fixed step size is longer than ours. The overall convergence time of our algorithms for a medium sized network should range within a few seconds with the same assumption in [15].

\section{CONCLUSiOnS}

In this paper, we have investigated the characteristics of fair allocations in presence of physical layer capture. The effect of physical layer capture has not been fully covered in literature on fair scheduling. We present a general model of physical layer channel and show that the feasible region of allocation vectors is non-convex in presence of physical layer capture.
In observation that the previous fair scheduling algorithms may not achieve optimal allocations, a new algorithm for log-utility fairness is present to determine the attempt probabilities of nodes. We first prove the concavenss of general utility on the domain of attempt probability and clarify that for log-utility fairness, the optimal attempt probability is given by the interference effect, defined as how much the transmissions of neighbors are degraded by the node. To run in a fully distributed way, we design the new scheduling algorithm such that it runs at each node with information locally available within the one-hop neighborhood.

As a result of the accuracy of our algorithm, the minimum node throughput as well as the aggregate utility is boosted up by 5 times on average. Furthermore, our algorithm prevents nodes from starvation unlike the previous algorithms discounting of dynamic-conflict graphs and causing starvation of nodes with weak links. Simulation results also show that the convergence time is less then 15 rounds with various numbers of node pairs in a $100 \times 100-\mathrm{m}^{2}$ area. The convergence time may be 
less than a few seconds for a median sized network.

\section{APPENDIX}

\section{A. Proof of Theorem 1}

We prove the theorem by showing that there exists a line segment joining a pair of allocation vectors that lies outside of the feasible region. Consider node $i$ and node $j$ and $k$ belonging to a minimum interference set $I$ of $i$. Without loss of generality, we assume that no nodes of $I$ have minimum interference sets such that the sets are included in $I$ and the size of the sets is greater than one. If any nodes have such sets, then we choose one of them and one minimum interference set for the node until the assumption is satisfied.

We first prove the theorem assuming that $\{i\}$ is not a minimum interference set of any nodes in $I$. Let $0<a<$ 1 and $X_{1}$ be a throughput vector obtained with $f_{i}=1$, $f_{k}=0$ and $f_{l}=a$ for all other node $l$ in $I$. Let $X_{2}$ be a throughput vector obtained with $f_{i}=1, f_{j}=0$ and $f_{l}=a$ for all other node $l$. Note that the throughput of a node becomes zero if its attempt probability equals zero. Let $X_{1}(i)$ be the node throughput of $i$ in vector $X_{1} . X_{1}(i)$ and $X_{2}(i)$ are one because either $j$ or $k$ of the minimum interference set does not transmit frames and node $i$ would experience no transmission errors.

Let $X$ be an allocation vector such that $X=\alpha X_{1}+$ $(1-\alpha) X_{2}$. Assume there exists an attempt probability vector $F\left(\ldots, f_{i}, \ldots, f_{j}, \ldots\right)$ achieving throughput allocation $X$. For any $\alpha>0, X(j), X(k)$ and $X(l)$ for all other node $l$ in $I$ must be positive because the attempt probabilities of the nodes in $I$ are in the range of 0 to 1 and node $i$ cannot interfere with the nodes in isolation. As a result, we have $f_{j}, f_{k}$ and $f_{l}>0$ and $q_{i}<1$. However, $X(i)$ must be 1 since $X_{1}(i)=X_{2}(i)=1$. No $F$ is feasible such that makes $X(i)$ equal to one and the feasible region is non-convex.

Now, let some of nodes in $I$ have $\{i\}$ as a minimum interference set. Assume $j$ is one of the nodes. If $j$ either interfere with or is interfered by some node $l$ in $I$, the non-convexity is proven by showing no attempt probabilities available for node $i$ and $j$ to have such a $X$ with $X_{1}$ from a probability vector $\left(f_{i}=1, f_{j}=\right.$ $\left.0, f_{l}=1\right)$ and $X_{2}$ from $\left(f_{i}=1, f_{j}=0, f_{l}=1\right)$. When $j$ would neither interfere nor be interfered, having $X_{1}$ from a vector $\left(f_{i}=1, f_{j}=0, f_{l}=a\right)$ for all other node $l, X_{2}$ from $\left(f_{i}=0, f_{j}=1, f_{l}=a\right)$ and $\alpha=1 / 2$, no attempt probabilities could be found for node $i$ and $j$ to make $X(j)=X(i)=1 / 2$.

\section{B. Proof of Theorem 2}

We prove the theorem by contradiction. Recall that the node throughput is given by Equation 1. It is simply shown that for any node $i$, if any neighbor node $j$ belonging in node $i$ 's minimum interference sets reduces attempt probability $f_{j}$, throughput $x_{i}$ increases. Now, assume a max-min fair scheduling algorithm produces $x_{i}$ and $x_{j}$, and $x_{i}<x_{j}$. By the lexicographic definition of the max-min fairness [32], decreasing $x_{j}$ could not help increase $x_{i}$ after fair scheduling finishes. However, since $x_{j}$ is in proportion of $f_{j}, x_{i}$ could increase and equal to a new value of $x_{j}$ by changing $f_{j}$. This contradicts the assertion that the scheduling algorithm achieves the max-min fairness and we have $x_{i} \geq x_{j}$.

\section{Proof of Theorem 3 and Corollary 1}

Let $S$ be the aggregate log utility of attempt probability vector $F$. That is, $S(F)=\sum_{i} \log \left(f_{i}\right)+\sum_{i} \log \left(q_{i}\right)$, where $F=\left(\ldots, f_{i}, \ldots\right)$. We prove the concaveness by showing the following concave inequality is preserved for all $F_{1}$ and $F_{2}$ and $\alpha \in[0,1]$ :

$$
S\left(\alpha F_{1}+(1-\alpha) F_{2}\right) \geq \alpha S\left(F_{1}\right)+(1-\alpha) S\left(F_{2}\right),
$$

We use the theorem that the sum of concave functions is concave. Since the aggregate $\log$ utility $S$ is $\sum_{i} \log \left(f_{i}\right)+\sum_{i} \log \left(q_{i}\right)$, the concaveness is proven if $\log \left(f_{i}\right)$ and $\log \left(q_{i}\right)$ are concave functions of attempt probability vector $F$.

First, $\log \left(f_{i}\right)$ is trivially a concave function. Now, consider $q_{i}=\sum_{J} c_{i}(J) \prod_{j} f_{j} \prod_{k}\left(1-f_{k}\right)$. Assuming $F_{3}=\alpha F_{1}+(1-\alpha) F_{2}$, we can show that $q_{h}\left(F_{3}\right)=$ $\alpha q_{h}\left(F_{1}\right)+(1-\alpha) q_{h}\left(F_{2}\right)$ since $q_{h}$ is a linear function of $f_{i}$. Let $Q_{h}(F)=\log \left(q_{h}(F)\right)$. the following inequality must be satisfied for $Q_{h}$ to be concave:

$$
q_{h}\left(F_{3}\right) \geq q_{h}\left(F_{1}\right)^{\alpha} \times q_{h}\left(F_{2}\right)^{(1-\alpha)} .
$$

Since $A^{\alpha} B^{(1-\alpha)} \leq \alpha A+(1-\alpha) B$ for all $A, B>0$, the right side of Inequality 8 is less than or equal to $\alpha q_{h}\left(F_{1}\right)+(1-\alpha) q_{h}\left(F_{2}\right)$, which is $q_{h}\left(F_{3}\right)$. Thus, $\log \left(q_{i}\right)$ is concave and the aggregate log utility is also concave.

Now, we prove the general utility function is also concave. For a given probability vector $F$, let $G_{i}(F)=$ $1 / x_{i}^{v}$ and $H_{i}(F)=\log \left(G_{i}(F)\right)=-v \log \left(x_{i}\right)$. Since $\log \left(x_{i}\right)$ is a concave function of $F, H_{i}$ is convex and we have the following inequality:

$$
\alpha \log \left(G_{i}\left(F_{1}\right)\right)+(1-\alpha) \log \left(G_{i}\left(F_{2}\right)\right) \geq \log \left(G_{i}\left(F_{3}\right)\right) .
$$

The left side of Inequality 9 is less than or equal to $\log \left(\alpha G_{i}\left(F_{1}\right)+(1-\alpha) G_{i}\left(F_{2}\right)\right)$ since $A^{\alpha} B^{(1-\alpha)} \leq$ $\alpha A+(1-\alpha) B$. We have $\alpha G_{i}\left(F_{1}\right)+(1-\alpha) G_{i}\left(F_{2}\right) \geq$ $G_{i}\left(F_{3}\right)$ and $G_{i}$ is convex. Thus, the general utility function, $\sum_{i}-G_{i}$ is the sum of concave functions and the utility function is also concave. 


\section{Proof of Theorem 4}

We show the outline of our proof. Consider an attempt probability vector $F_{1}=\left(\ldots, f_{i}, \ldots\right)$. Let $F_{2}$ be a probability vector after updating $f_{i}$ to satisfy the condition where the partial derivative is zero. From the concaveness of aggregate general utilities, we have the following inequality:

$$
S\left(F_{2}\right)-S\left(F_{1}\right) \geq \nabla S\left(F_{2}\right)\left(F_{2}-F_{1}\right),
$$

where $S$ is an aggregate utility function of probability vectors. Let $F(i)$ be the attempt probability of node $i$ in vector $F$. Since only $f_{i}$ has been updated, $F_{2}(j)-F_{1}(j)$ is zero for all other $j$. Furthermore, $\partial S / \partial f_{i}$ is equal to zero at the updated vector $F_{2}$. We have $\nabla S\left(F_{1}\right)\left(F_{2}-\right.$ $\left.F_{1}\right)=0$ and $S\left(F_{2}\right) \geq S\left(F_{1}\right)$. Thus, updating the attempt probability improves the aggregate utility and the maximum finally will be reached by repeating this.

\section{REFERENCES}

[1] A. Kochut, A. Vasan, A. U. Shankar, and A. Agrawala, "Sniffing out the correct physical layer capture model in $802.11 \mathrm{~b}$," in ICNP 2004, 2004.

[2] M. M. Carvalho and J. J. Garcia-Luna-Aceves, "A scalable model for channel access protocols in multihop ad hoc networks," in MobiCom '04. ACM Press, 2004, pp. 330-344.

[3] H. S. Chhaya and S. Gupta, "Performance modeling of asynchronous data transfer methods of IEEE 802.11 MAC protocol," Wirel. Netw., vol. 3, no. 3, pp. 217-234, 1997.

[4] M. Garetto, T. Salonidis, and E. W. Knightly, "Modeling perflow throughput and capturing starvation in CSMA multi-hop wireless networks," in IEEE INFOCOM 2006, April 2006.

[5] M. Soroushnejad and E. Geraniotis, "Probability of capture and rejection of primary multiple access interference in spread spectrum networks," in IEEE Transactions on Communications, vol. 39, no. 6, 1991, pp. 986-994.

[6] T. Nandagopal, T.-E. Kim, X. Gao, and V. Bharghavan, "Achieving MAC layer fairness in wireless packet networks," in MobiCom '00. ACM Press, 2000, pp. 87-98.

[7] K. Kar, S. Sarkar, and L. Tassiulas, "Achieving proportional fairness using local information in aloha networks," IEEE Transaction on Automatic Control, vol. 49, no. 10, pp. 18581862, October 2004.

[8] H. Chang, V. Misra, and D. Rubenstein, "Throughput and fairness in CSMA/CA wireless networks," Columbia University, Tech. Rep., 2006.

[9] C. Namislo, "Analysis of mobile radio slotted ALOHA networks," IEEE Journal on Selected Areas in Communications, vol. SAC-2, pp. 199-204, August 1984.

[10] I. Cidon and M. Sidi, "The effect of capture on collisionresolution algorithms," IEEE transaction on communications, vol. COM-33, 4, pp. 317-324, 1985.

[11] R. Nelson and L. Kleinrock, "The spatial capacity of a slotted ALOHA multihop packet radio network with capture," IEEE Transactions on Communications, vol. 32, pp. 684-694, 1984.

[12] F. Baccelli, B. Blaszczyszyn, and P. Muhlethaler, "An Aloha protocol for multihop mobile wireless networks," IEEE Trans. on Information Theory, vol. 52, no. 2, pp. 421-436, 2006.

[13] H. Chang, V. Misra, and D. Rubenstein, "A general model and analysis of physical layer capture in 802.11 networks," in IEEE INFOCOM 2006, April 2006.
[14] F. Kelly, A. Maulloo, and D. Tan, "Rate control in communication networks: shadow prices, proportional fairness and stability," in Journal of the Operational Research Society, vol. 49, 1998.

[15] X. Wang and K. Kar, "Cross-layer rate control for end-to-end proportional fairness in wireless networks with random access," in MobiHoc '05. New York, NY, USA: ACM Press, 2005, pp. 157-168.

[16] _ , "Distributed approaches for proportional and max-min fairness in random access adhoc networks," in 40th Annual Conference on Information Sciences and Systems (CISS), 2006.

[17] X. L. Huang and B. Bensaou, "On max-min fairness and scheduling in wireless ad-hoc networks: analytical framework and implementation." in MobiHoc '01. ACM, October 2001, pp. 221-231.

[18] L. Tassiulas and S. Sarkar, "Maxmin fair scheduling in wireless networks," in INFOCOM 2002, 2002.

[19] J. Arnbak, "Capacity of slotted aloha in rayleigh fading channels," IEEE Journal On Selected Areas in Communications, vol. SAC-5, pp. 261-269, February 1987.

[20] D. Davis and S. Gronemeyer, "Performance of slotted aloha random access with delay capture and randomized time of arrival," IEEE Transactions on Communications, vol. COM-28, pp. 703-710, 1980.

[21] K. Cheun and S. Kim, "Joint delay-power capture in spreadspectrum packet radio networks," IEEE Transaction on Communications, vol. 46, no. 4, pp. 450-453, 1998.

[22] C. Reis, R. Mahajan, M. Rodrig, D. Wetherall, and J. Zahorjan, "Measurement-based models of delivery and interference in static wireless networks," in SIGCOMM '06: Proceedings of the 2006 conference on Applications, technologies, architectures, and protocols for computer communications. New York, NY, USA: ACM Press, 2006, pp. 51-62.

[23] L. Chen, S. H. Low, M. Chiang, and J. C. Doyle, "Cross-layer congestion control, routing and scheduling design in ad hoc wireless networks," in IEEE INFOCOM 2006, April 2006.

[24] H. Chang, V. Misra, and D. Rubenstein, "Throughput and fairness in random access networks," Columbia University, Tech. Rep. CUCS-033-06, 2006.

[25] B. Radunovic and J.-Y. L. Boudec, "Rate performance objectives of multihop wireless networks," IEEE Transactions on Mobile Computing, vol. 3, no. 4, pp. 334-349, October 2004.

[26] L. Massouli and J. Roberts, "Bandwidth sharing: Objectives and algorithms," IEEE Transaction on Networking, vol. 10, no. 3, pp. 320-328, June 2002.

[27] W. S. Massey, Algebraic Topology: An Introduction. SpringerVerlag, 1977, vol. 56.

[28] H. Chang, V. Misra, and D. Rubenstein, "Linear approximation of optimal attempt rate in random access networks," Columbia University, Tech. Rep. CUCS-032-06, 2006.

[29] M. Takai, J. Martin, and R. Bagrodia, "Effects of wireless physical layer modeling in mobile ad hoc networks," in MobiHoc '01: Proceedings of the 2nd ACM international symposium on Mobile ad hoc networking \& computing. New York, NY, USA: ACM Press, 2001, pp. 87-94.

[30] D. P. Bertsekas, Nonlinear Programming, 2nd ed. Athena Scientific Press, 1999.

[31] R. Jain, D. Chiu, and W. Hawe, "A quantitative measure of fairness and discrimination for resource allocation in shared computer systems," Digital Equipment Corporation, Maynard, MA, USA, DEC Research Report TR-301, September 1984.

[32] D. Bertsekas and R. Gallager, Data Networks. Prentice Hall, 1992. 